\title{
The rich frequency spectrum of the triple-mode variable AC And
}

\author{
Géza Kovács ${ }^{1,2}$, Gáspár A. Bakos ${ }^{3}$ and Joel D. Hartman ${ }^{3}$ \\ ${ }^{1}$ Dept. of Physics \& Astrophysics, U. North Dakota, Grand Forks, ND \\ ${ }^{2}$ Konkoly Obs., Budapest, Hungary \\ ${ }^{3}$ Princeton University, Department of Astrophysical Sciences, Princeton
}

\begin{abstract}
Fourier analysis of the light curve of AC And from the HATNet database reveals the rich frequency structure of this object. Above 30 components are found down to the amplitude of 3 mmag. Several of these frequencies are not the linear combinations of the three basic components. We detect period increase in all three components that may lend support to the Population I classification of this variable.
\end{abstract}

Keywords. stars: variables, Cepheids, fundamental parameters

There are a handful of objects that seem to exhibit sustained pulsations in three radial modes (Wils et al. 2008). These are important objects with respect to the unique opportunity to derive their basic physical parameters (i.e., mass, luminosity and temperature) by using their periods only (assuming that the metallicity is known and that linear pulsation periods are close to the observed [nonlinear] ones - see Kovács \& Buchler (1994) and Moskalik \& Dziembowski (2005)).

By using the 9600 datapoint light curve gathered by HATNet $\dagger$, we perform a Fourier frequency analysis on AC And, the prototype of radial three-mode pulsators. The analysis of the basic photometric data yields the three modes and their low-order linear combinations with high $\mathrm{S} / \mathrm{N}$. To reach the millimagnitude level, we need to minimize systematics. Because of the high amplitudes of the low-order components, we cannot use TFA (Kovács et al. 2005) in the frequency search mode, since it is based on the low S/N assumption. Therefore, we opted to use the method of Kovács \& Bakos (2008). Here the most reliably determined signal components and the TFA template light curves are simultaneously fitted. After cleaning the data by the so-obtained TFA filter, we get clean, successively prewhitened spectra as shown on the left panel of Fig. 1. Successive prewhitening leads to the identification of many components, most of which are clearly the linear combinations of the 3 basic frequencies. However, both the frequency spectra (top right panel of Fig. 1) and the decomposed individual modes show the presence of non-fitting components even when considering linear combinations of the main frequencies up to order 10. This feature has also been noted in the discovery paper of Fitch \& Szeidl (1976).

We also examined the rate of period change during the past 55 years. Knowledge of the period change is important, because if it is due to stellar evolution, it may help to select the right model if other parameters are ambiguous. From the analysis of Guman (1981, 1982) it seems that all three modes exhibit period increases much higher than expected

$\dagger$ The Hungarian-made Automated Telescope Network (Bakos et al. 2004) consists of 6 wide field-of-view, small aperture autonomous telescopes located in Hawaii (Mauna Kea) and Arizona (Fred Lawrence Whipple Observatory). The prime purpose of the project is to search for extrasolar planets via transit technique. 

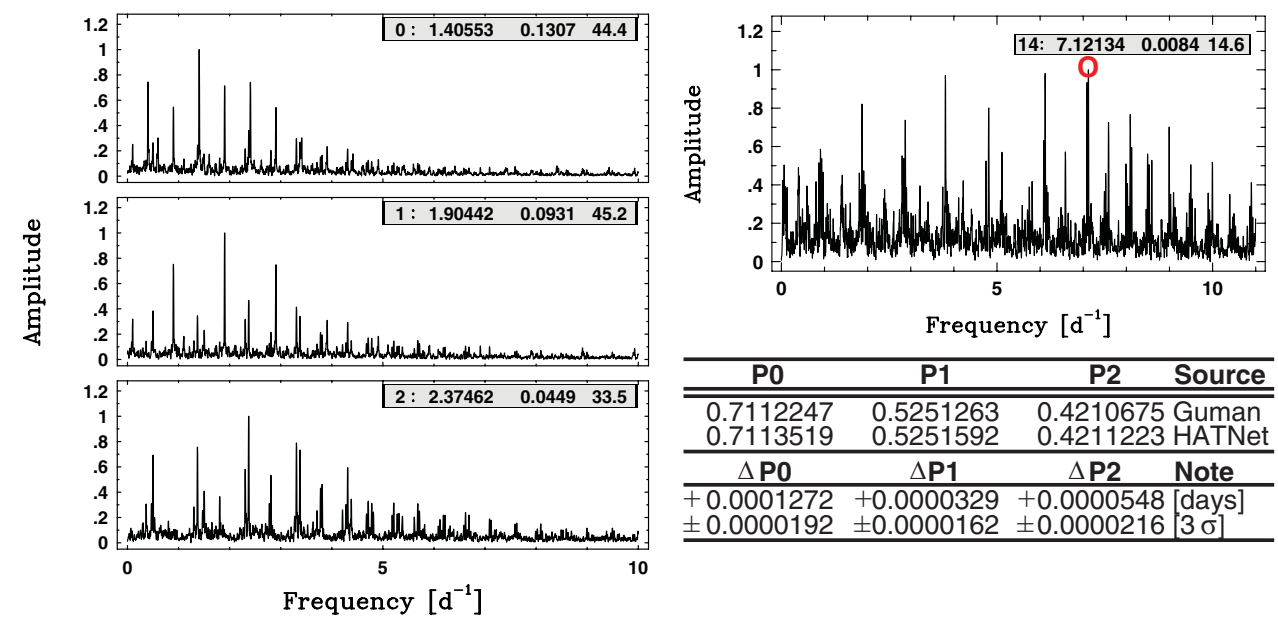

Figure 1. Left: Successively prewhitened frequency spectra of AC And; insets: prewhitening order; peak frequency $\left[\mathrm{d}^{-1}\right]$, amplitude $[\mathrm{mag}], \mathrm{S} / \mathrm{N}$. Top right: Frequency spectrum of AC And after the 14th prewhitening. The circled peak corresponds to one of the handful of components that cannot be identified as the linear combination of the three main frequencies. Bottom right: Periods detected 55 years apart (upper two rows) and their differences together with the corresponding errors (lower two rows).

from an RR Lyrae star (Kovács \& Buchler 1994). As seen in the table section of Fig. 1, the periods derived from the HATNet data confirm the period increase during the past 55 years. Note however that the speed of increase derived here is $\sim 4$ times higher than given by Jurcsik et al. (2006) based on earlier data.

Except perhaps for the Strömgren photometry of Peña et al. (2005), AC And lacks good multicolor time series observations and deep spectroscopic work. As a result, the physical parameters of the star are still poorly known. Opposite to Peña et al. (2005), we think that the available photometric and pulsation data support that $\mathrm{AC}$ And is a relatively high $\left(\sim 3 M_{\odot}\right)$ mass star with $T_{\text {eff }} \approx 5800 \pm 200 \mathrm{~K}, \log g \approx 2.5 \pm 0.5$ and $[\mathrm{Fe} / \mathrm{H}]$ $\approx-0.5 \pm 0.5$.

Acknowledgement: G. K. thanks the Hungarian Scientific Research Foundation (OTKA) for support through grant K-81373.

\section{References}

Bakos, G. A., Noyes, R. W., Kovács, G., Stanek, K. Z., Sasselov, D. D., \& Domsa, I. 2004, $P A S P, 116,266$

Fitch, W. S. \& Szeidl, B. 1976, ApJ, 203, 616

Guman, I. 1981, IBVS, 2046

Guman, I. 1982, Comm. Konkoly Obs., 78, 1

Jurcsik, J., Szeidl, B., Váradi, M., et al. 2006, A\&A, 445, 617

Kovács, G. \& Bakos, G. A. 2008, CoAst, 157, 82

Kovács, G. \& Buchler, J. R. 1994, A\&\&A, 281, 749

Kovács, G., Bakos, G. A., \& Noyes, R. W. 2005, MNRAS, 356, 557

Moskalik, P. \& Dziembowski, W. A. 2005, A $\mathscr{E} A$, 434, 1077

Peña, J. H., Peniche, R., Hobart, M. A., de La Cruz, C., \& Gallegos, A. A. 2005, Rev. Mexicana AyA, 41, 461

Wils, P., Rozakis, I., Kleidis, S., Hambsch, F.-J., \& Bernhard, K. 2008, A\&̊A, 478, 865 